\title{
Deep sequencing of multiple regions of glial tumors reveals spatial heterogeneity for mutations in clinically relevant genes
}

\author{
Akash Kumar $^{1 *}$, Evan A Boyle ${ }^{1}$, Mari Tokita ${ }^{2,3}$, Andrei M Mikheev ${ }^{3}$, Michelle C Sanger ${ }^{4}$, Emily Girard ${ }^{4}$, John R Silber ${ }^{3}$, \\ Luis F Gonzalez-Cuyar ${ }^{5}$, Joseph B Hiatt ${ }^{1}$, Andrew Adey ${ }^{1}$, Choli Lee ${ }^{1}$, Jacob O Kitzman ${ }^{1}$, Donald E Born" \\ Daniel L Silbergeld ${ }^{3}$, James M Olson ${ }^{4}$, Robert C Rostomily ${ }^{3^{*}}$ and Jay Shendure ${ }^{\text {* }^{*}}$
}

\begin{abstract}
Background: The extent of intratumoral mutational heterogeneity remains unclear in gliomas, the most common primary brain tumors, especially with respect to point mutation. To address this, we applied single molecule molecular inversion probes targeting 33 cancer genes to assay both point mutations and gene amplifications within spatially distinct regions of 14 glial tumors.

Results: We find evidence of regional mutational heterogeneity in multiple tumors, including mutations in TP53 and RB1 in an anaplastic oligodendroglioma and amplifications in PDGFRA and KIT in two glioblastomas (GBMs). Immunohistochemistry confirms heterogeneity of TP53 mutation and PDGFRA amplification. In all, 3 out of 14 glial tumors surveyed have evidence for heterogeneity for clinically relevant mutations.

Conclusions: Our results underscore the need to sample multiple regions in GBM and other glial tumors when devising personalized treatments based on genomic information, and furthermore demonstrate the importance of measuring both point mutation and copy number alteration while investigating genetic heterogeneity within cancer samples.
\end{abstract}

\section{Background}

Regional heterogeneity of mutations has been observed in a variety of tumor types $[1,2]$. This intratumoral heterogeneity has broad implications for the clinical management of cancer patients, especially in the current paradigm of personalized medicine based on genomic analysis of a single cancer biopsy. Within the context of primary brain tumors, several groups have previously identified heterogeneity of gene amplifications in genes EGFR and PDGFRA in glioblastoma multiforme (GBM) using fluorescence in situ hybridization (FISH) and array-comparative genomic hybridization on multiple regions within primary tumors $[3,4]$. Despite the

\footnotetext{
*Correspondence: akash1@uw.edu; rosto@u.washington.edu; shendure@u. washington.edu

'Department of Genome Sciences, University of Washington, Seattle, WA 98195, USA

${ }^{3}$ Department of Neurosurgery, University of Washington, Seattle, WA 98195 , USA

Full list of author information is available at the end of the article
}

dropping cost of DNA sequencing, however, the extent of point mutational heterogeneity in brain tumors remains limited to a single case of GBM [5]. This is in part because the investigation of intratumoral heterogeneity requires both sampling and deep sequencing of multiple regions in a tumor.

We recently developed a method to identify low frequency mutations across known cancer genes [6] using the single molecule molecular inversion probe (smMIP) assay, which combines multiplex target capture with single molecule tagging [6,7]. Here, we extend this technique to detect gene amplifications and examine intratumoral heterogeneity by targeting 33 cancer genes across 62 spatial sections of 14 glial tumors, including 10 grade IV gliomas (all GBMs), three grade III gliomas (one each of ependymoma, astrocytoma, and anaplastic oligodendroglioma) and one grade II astrocytoma. We detected intratumoral heterogeneity in both point mutations and amplifications of genes implicated as glioma tumor drivers and therapeutic targets. 


\section{Results}

\section{Study design}

To assess heterogeneity within gliomas, we dissected each of 14 tumors into 3 to 5 regions per tumor (Figure 1A; Table S1 in Additional file 1). We used the smMIP assay on genomic DNA isolated from each region to identify single nucleotide variants and high level copy amplifications (Figure 1B; Figure S1 in Additional file 1). smMIP probes capture target sequence into covalently linked circular molecules after polymerase extension and ligation. Following barcoding-PCR, sample pooling, sequencing, deduplication and alignment, we identified high level amplifications and point mutations (Figure 1B,C; Figure S1 in Additional file 1).
Across the 14 tumors and 33 genes considered in this analysis, we identified a total of 33 putative proteinaltering mutations (Tables S1 and S2 in Additional file 1). Tumors had between zero and 16 putative proteinaltering mutations, with a median of two. TP53 was the most commonly mutated gene, with mutations found in 8 out of 14 tumors (Figure 2A; Table S3 in Additional file 1). One tumor, BI12, had many more candidate somatic mutations than other tumors $(\mathrm{n}=16$ versus median $\mathrm{n}=2$ in other tumors). Mutations in this GBM were predominantly $\mathrm{G}>\mathrm{T}$ (or $\mathrm{C}>\mathrm{A}$ ) transversions (8 of 16 total), possibly representing mutation from unrepaired 8-oxo-guanine damage. Most mutations were observed across all tumor regions of BI12, consistent with a defect

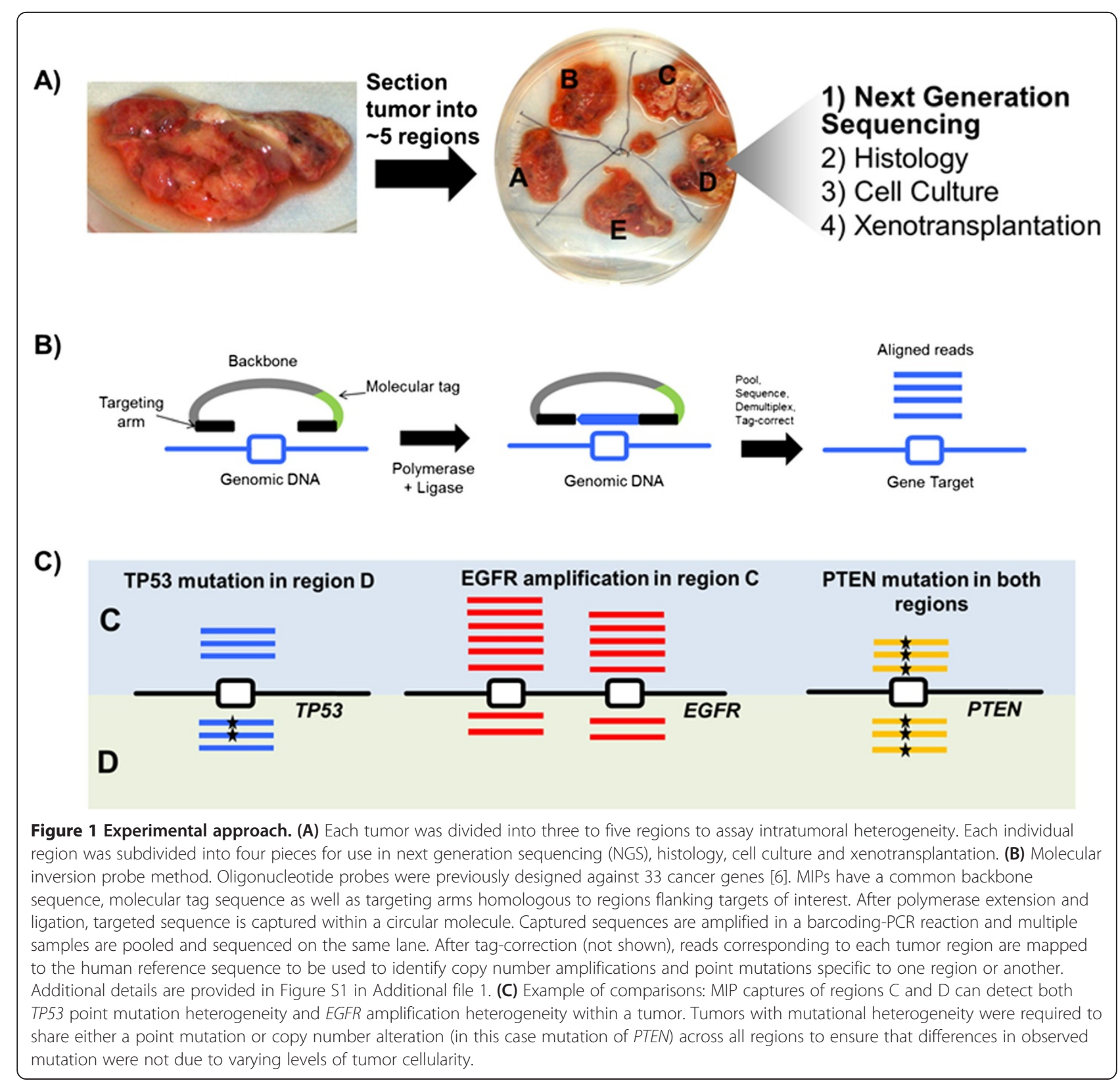




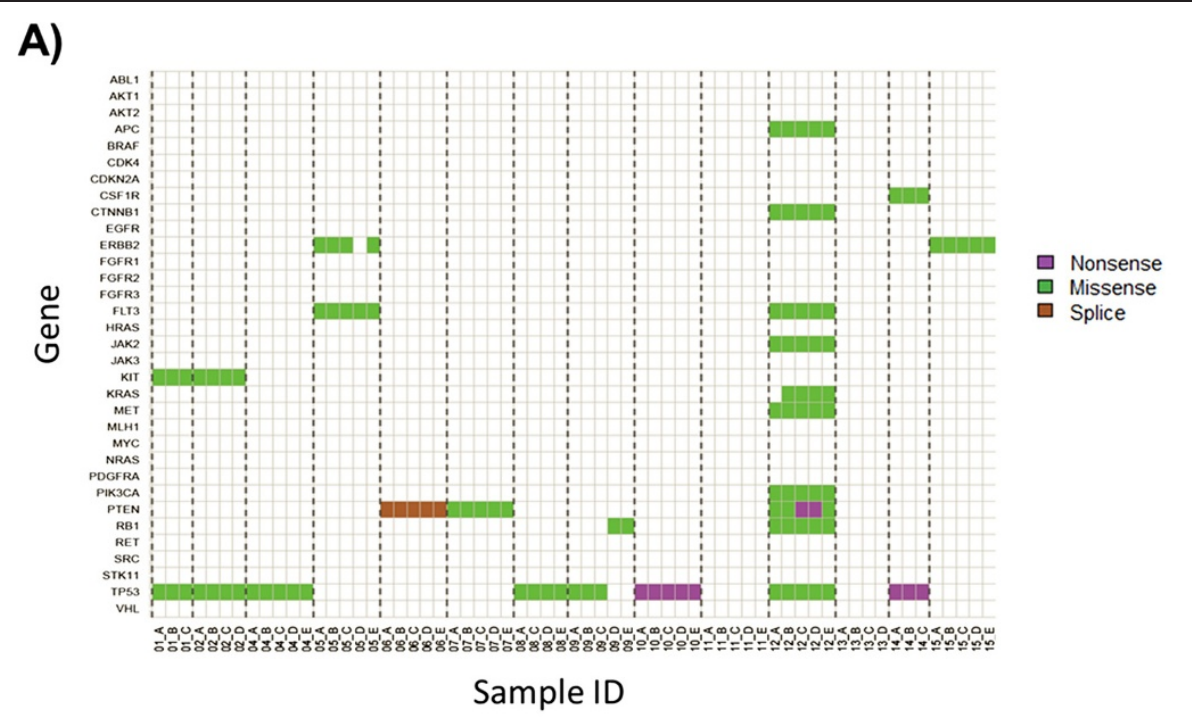

B)

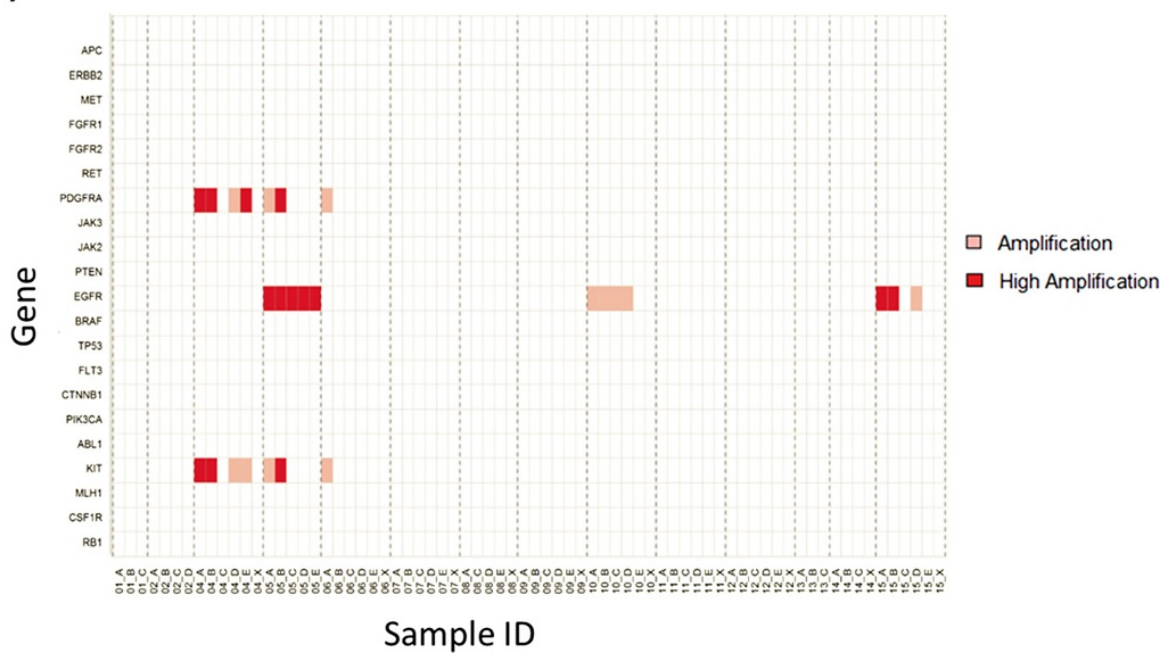

Figure 2 Summary of heterogeneity observed across all samples. (A) Protein-altering mutations detected across all tumor regions. Genes mutated twice in the same tumor region are not identified here but can be found within a table of all mutations (Table S3 in Additional file 1). (B) High level gene amplifications detected by smMIP assay. Copy number was estimated by comparing all tumor samples against $12 \_X$, a universal control from Bl12 (see Figure S2 in Additional file 1 for analysis using patient matched controls). 'Amplification' indicates genes with coverage three-fold higher than median coverage across a sample. 'High Amplification' indicates genes with coverage six-fold higher than median coverage across a sample. Region X refers to brain tissue grossly uninvolved by tumor. Our approach would miss any low-level gene amplifications within these tumors.

in DNA repair arising early in the development of the tumor.

To identify high level gene amplifications in tumors, we compared read depth of smMIP-targeted regions in each tumor against that of a control tissue. As smMIP sequencing suggested that a subset of control tissues were contaminated with tumor cells, we performed analyses using either patient-matched controls (Figure S2 in Additional file 1) or a 'universal' control (Figure 2B). For the latter we selected control tissue from tumor BI12, as it appeared to have the least tumor contamination based on allele fraction of known pathogenic point mutations, and restricted copy number analyses to targets with $>30 \times$ coverage in control tissue from BI12 as well as targets whose GC percentage ranged from 30 to $60 \%$ ( $n=885$ capture probes). A careful review of discrepant calls when using patient-matched versus a universal control indicated that use of the universal control was more sensitive in identifying bona fide amplification events (as confirmed with Taqman assays) secondary to the contamination of a subset of control tissues with tumor cells. After applying our filters (see Materials and methods), a total of 21 genes could be assayed in a total of 62 regions across 14 tumors (Figure 2B). 
The ratio of coverage of each probe was calculated relative to the control tissue (from BI12). We used DNACopy [8] to segment genes and obtain $R$, the mean ratio of coverage relative to control for each gene. We estimated the copy number for each gene by dividing $\mathrm{R}$ for each gene by the median value of $\mathrm{R}$ across all genes for each tissue. Genes with ratios above 3 were called as amplified. Genes with ratios above 6 were called as highly amplified. We did not measure deletion of genes using this method.

This process identified five tumors with gene amplifications, with three having one or more regions with a highly amplified gene (Figure 2B). Three tumors had amplification of both PDGFRA and KIT, and three tumors had amplification of EGFR. We validated copy number estimates for a subset of calls using a variety of different methods, including Taqman quantitative PCR (qPCR; across all tumors for EGFR and tumors BI05, BI06 and BI15 for PDGFRA), as well as whole genome sequencing (in tumor BI15 for EGFR). MIP copy number estimates of EGFR were highly correlated $\left(R^{2}=0.90\right)$ with delta $\mathrm{Ct}$ obtained by Taqman qPCR when compared across all 62 regions sequenced (Figure S3 in Additional file 1). Additionally for five tumor regions of BI15 that were subjected to light-whole genome sequencing, EGFR copy number estimates were consistent between whole genome sequencing and smMIP techniques (Supplementary methods and Figure S4 in Additional file 1).

Tumors in which only a subset of regions possess an amplification or point mutation with no other mutation shared across regions can be the result of either mutational heterogeneity within a tumor or varying levels of tumor content between different tumor regions. As an example, tumor BI15 was called as amplified for EGFR in two out of five regions with no other somatic mutations/point mutations detected across the tumor (Figures S5 and S6 in Additional file 1). Upon close inspection of histologic slides prepared from adjacent tissue, the observed difference in amplification was most likely due to lower tumor cellularity within other regions of this tumor rather than intratumoral genetic heterogeneity. This was also seen in tumor BI04, where one region without detectable PDGFRA amplification also had lower frequencies of a TP53 mutation seen across all regions. For this reason, we chose to restrict our interpretation of intratumoral heterogeneity to tumors in which all regions also shared a point mutation or gene amplification. Three tumors met these criteria and are described below.

\section{Spatial heterogeneity of TP53 and RB1 point mutations}

One tumor exhibited clear spatial heterogeneity with respect to point mutations within the 33 genes investigated (Figure 3). BI09, an IDH1-mutant anaplastic oligodendroglioma, had a high allele fraction ( $>30 \%$ reads supporting mutation) inactivating mutation ( $\mathrm{R} 248 \mathrm{H})$ in TP53 in only two regions of the tumor (A and $\mathrm{B}$ ). This tumor had high allele fraction mutations in $R B 1$ exclusively in two other regions (D and E) within the same tumor. Both TP53 and RB1 mutations were present at trace levels $(<1 \%)$ within region $\mathrm{C}$. As clinical workup indicated that $\mathrm{BI} 09$ had an IDH1 mutation, we investigated all regions of this tumor by Sanger sequencing and found that regions $\mathrm{A}$ to $\mathrm{E}$

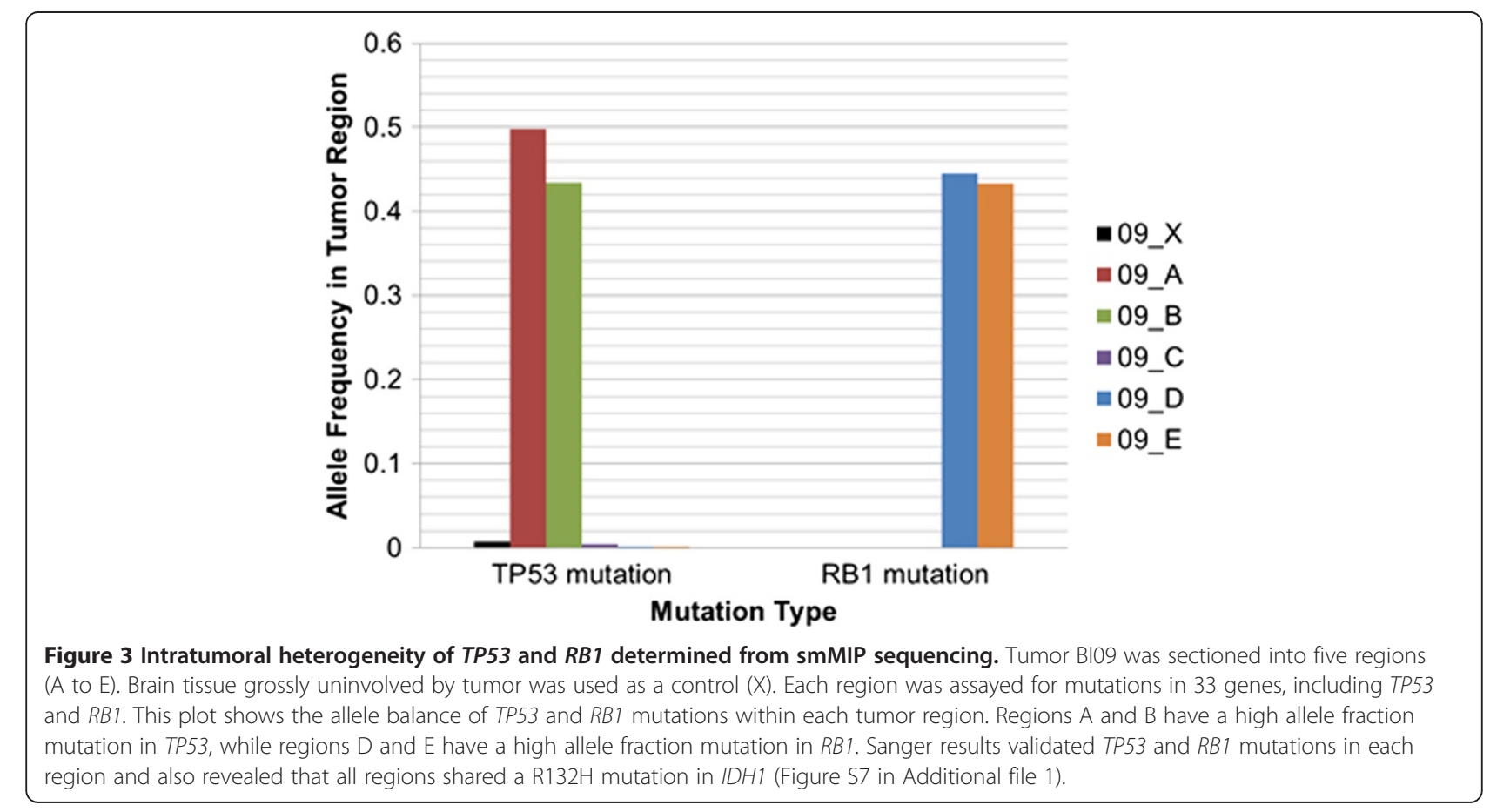


shared the IDH1 R132H mutation. Sanger sequencing also validated the TP53 mutation in regions $A$ and B as well as the RB1 mutation in regions D and E (Figure S7 in Additional file 1). Immunohistochemistry of p53 and IDH1-R132H expression on tissue adjacent to regions $\mathrm{A}$ to E provided additional confirmatory evidence (Figure S8 in Additional file 1). These findings are consistent with an IDH1-mutant tumor subsequently diverging to form subclones with mutations in RB1 and TP53 [9,10]. A neuropathologist (LFG-C) scored the grade and diagnosis for each of these samples blinded to the mutation type. Interestingly, the presence of TP53 mutation correlated with the higher grade histology (Table S4 in Additional file 1). The clinical significance is unknown but this serves as a potential example of how genomic heterogeneity may affect histology of a tumor.

\section{Spatial heterogeneity of PDGFRA and KIT amplifications} Our smMIP technique detected amplification of PDGFRA, $K I T$ and EGFR within tumor BI05, an IDH1-wild type glioblastoma. In this tumor EGFR amplification was seen across all tumor regions, while amplification of both PDGFRA and KIT was detected in two of five regions (Figure 4A). As KIT is located near PDGFRA on chromosome 4, shared amplification of these genes is expected [11]. Taqman real-time PCR assays performed in quadruplicate confirmed both the amplification in EGFR and the amplification in PDGFRA across all assayed regions
(Figure 4B). Immunohistochemistry of PDGFRA and EGFR on tissue adjacent to regions $\mathrm{A}$ to $\mathrm{E}$ provided additional confirmatory evidence (Figure S9 in Additional file 1).

Similarly, we detected heterogeneity of PDGFRA amplification within BI06, an IDH1-mutant glioblastoma. This tumor had amplification of PDGFRA and KIT in region A not detected within other regions (Figure 5A). Taqman qPCR confirmed amplification of region A, mild amplification in region $\mathrm{B}$ and no amplification in regions $\mathrm{C}, \mathrm{D}$ and $\mathrm{E}$ (Figure $5 \mathrm{~B}$ ). All other regions of this tumor had somatic mutations in PTEN, such that reduced tumor cellularity is an unlikely explanation for our observations.

\section{Additional cases of heterogeneity are potential passenger mutations}

A missense mutation in KRAS was observed at moderate allele fraction ( $10 \%$ of reads) in region $\mathrm{D}$ of the glioblastoma BI12 and was not detected in at least one other region (Table S3 in Additional file 1). As this mutation does not occur within known mutation hotspots and is in a tumor with signs of hypermutation (BI12), the clinical significance of this heterogeneity remains unclear. Other somatic point mutations are heterogeneous across an individual tumor but occur within genes that have another, ubiquitously distributed mutation. BI12 has missense mutations in PTEN that are observed in regions $\mathrm{A}, \mathrm{B}$ and $\mathrm{C}$ and not in region $\mathrm{D}$ (Table $\mathrm{S} 3$ in Additional file 1). This tumor also has another high allele fraction mutation in

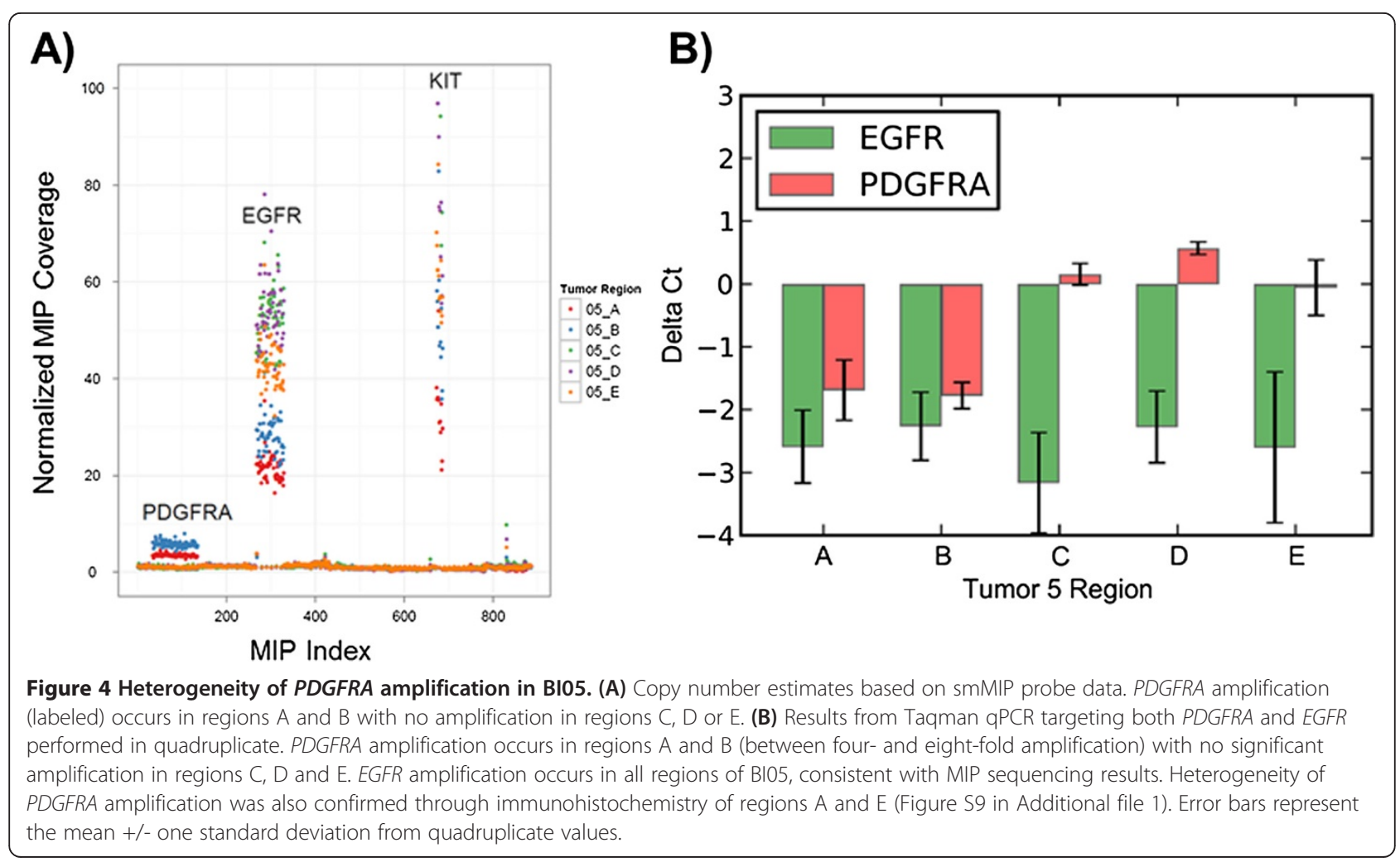




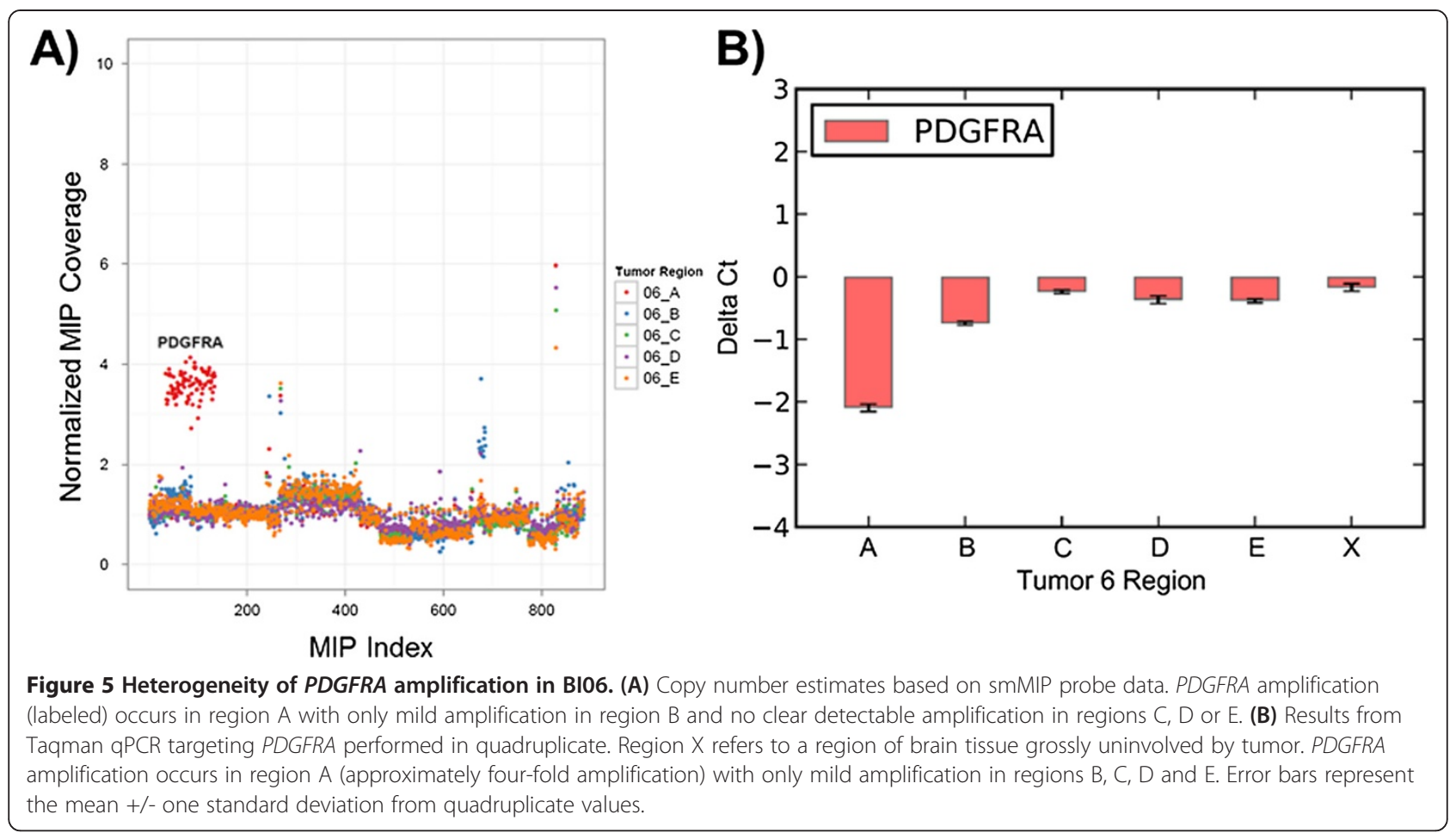

this gene that is present across all regions of this tumor. A similar scenario is seen in the astrocytoma BI08. Regions $\mathrm{D}$ and $\mathrm{E}$ of this tumor have low allele fraction point mutations in TP53, but all regions share another high allele fraction mutation in the same gene. These results suggest that the heterogeneously observed mutations observed are more likely secondary passenger mutations that occurred after PTEN and TP53 were inactivated in tumors BI12 and BI08, respectively (Table S3 in Additional file 1). An alternative possibility is that tumors may contain distinct subpopulations in which genes were inactivated by a different mutation.

\section{Discussion}

These results demonstrate that intratumoral spatial heterogeneity with respect to clinically relevant genes occurs among multiple types of brain tumors, and spans the mutational spectrum from copy number to point mutations. Across a set of recurrently mutated cancer genes (33 genes examined for point mutations, 21 genes for amplifications), we observed heterogeneity for clinically relevant mutations in 3 of 14 (21\%) glial tumors. These include point mutations in TP53 and RB1 as well as amplifications in PDGFRA/KIT. All cases of mutational heterogeneity that we detected in a tumor occur in adjacent regions, consistent with the hypothesis that spatially distinct regions represent divergent subclones of a single tumor.

Historically, in anaplastic oligodendroglioma with intact 1p, mutations in TP53 were found to stratify outcomes, with median survival of 71 versus 16 months in patients with mutant versus wild-type TP53, respectively [12]. While not specifically applying to our patient in whom $1 \mathrm{p} / 19 \mathrm{q}$ is deleted, our data demonstrating discrete differences in TP53 status from different regions within an individual tumor nevertheless show the potential of genetic heterogeneity to confound the assignment of prognostication based on the detection of specific molecular markers.

In addition, decision-making regarding the use of receptor tyrosine kinase inhibitors could be influenced by the status of amplifications/mutations in PDGFRA. Our finding of regional heterogeneity of PDGFRA within tumors BI05 and BI06 confirms recent work by Sottoriva et al. and others and suggests that a single biopsy may not be sufficient to allow for informed application of targeted therapies against these presumed oncogenic drivers $[3,4,13]$. Clinical decision-making at recurrence will also likely be impacted by regional heterogeneity. Nickel et al. [5] compared mutations within a group of 10 genes from 2 regionally distinct samples of a single GBM at initial resection and 2 subsequent recurrences. No heterogeneity was detected at initial resection but heterogeneity of PIK3CA and PTEN mutation was detected at the first recurrence, and heterogeneity of PIK3CA, TP53 and EGFR mutation was detected at the second recurrence.

This study used tumor-adjacent brain to serve as a 'control tissue' to help identify somatic mutations. However, after extracting DNA and investigating mutations, we observed that in several cases the grossly normal brain tissue actually contained a large fraction of infiltrating 
tumor cells. This aspect of our study ultimately complicated the process of calling somatic point mutations (requiring filtering against databases of germline variation) and copy number (necessitating our use of a universal control). While blood would be the most appropriate material to use as a control we did not have institutional review board approval to collect blood at the time these experiments were conducted (subsequently modified to allow for blood collection in the future).

In this study we were able to identify amplification of only a subset of genes of interest, as some genes had too few probes to accurately determine copy number. Our study also did not detect genomic rearrangements and deletions such as the EGFR VIII deletion commonly found in GBM. However, one can imagine expanding this assay to consider amplifications and deletions with smMIPs by tiling probes at higher density and incorporating known SNP positions to aid in identifying cases of loss of heterozygosity. One could also capture additional glioma-relevant genes like $I D H 1$ and $I D H 2$ by adding probes targeting these genomic regions.

Our investigation focused on regional heterogeneity within a tumor, instead of the microscopic heterogeneity that is likely present within a given tumor biopsy. As we performed the smMIP assay on DNA extracted from tissue pieces that likely contained millions of cells, we would likely miss cases of heterogeneity where only a small population of cells within a biopsy contained a mutation (such as an amplification). Use of techniques such as immunohistochemistry, FISH and, more recently, single cell sequencing remain necessary to characterize the extent of microscopic heterogeneity in tumors.

These results validate the smMIP approach as a scalable and cost-effective platform for deep sequencing of cancer genomes to examine subclonal variation. Despite deeply sequencing multiple sections of 14 tumors, our survey required only one lane of sequencing on the Illumina HiSeq because we focused on well-known gene targets of mutation in cancer. In contrast to the technique used by a similar investigation [5], our method is also easily scaled and amenable to automation with samples processed in 96well formats. This advantage in scalability enables one to easily assay many more regions (tens to hundreds) per tumor to obtain much finer scale pictures of intratumoral heterogeneity, as we are likely underestimating its extent even here by sampling of only a few regions. While our study represents an improvement over previous studies, analysis of greater number of genes in a greater number of tumors will be necessary to determine rates of regional heterogeneity in different driver mutations across GBMs.

\section{Conclusions}

We find multiple instances of regional heterogeneity in clinically relevant cancer genes within malignant gliomas at the time of diagnosis. We also demonstrate a scalable technique that can be used to efficiently characterize regional genetic heterogeneity for both point mutations and copy number alterations in tumors. Future challenges will include how best to interpret cases of intratumoral heterogeneity and test its impact in the context of clinical trials using targeted therapy approaches.

\section{Materials and methods \\ Samples}

Freshly resected brain tumor specimens from adult patients were obtained with informed consent as part of the Genomics Big Idea pilot program (UW/FHCRC). Tissue, patient demographics and final diagnosis were obtained in accordance with protocols approved by the institutional review board at the University of Washington. Tumors were divided into three to five regions, depending on size. Tissue from each region was then subdivided into four pieces for use in next generation sequencing, histology, cell culture and xenotransplantation (Figure 1). In 10 cases, brain grossly uninvolved by tumor was resected to provide adequate surgical access and was utilized as a source of germline or 'control' DNA to identify somatic mutations (Table S1 in Additional file 1). These tissues are referred to as regions " $\mathrm{X}$ " in this manuscript. For all samples, DNA was isolated from snap-frozen tissue pieces using the QIAGEN DNEasy Blood and Tissue kit (Qiagen, Venlo, Netherlands).

\section{Targeted capture and sequencing}

The smMIP assay was used to genotype candidate genes. Probes were previously designed by Hiatt et al. [6] against 33 genes that are commonly mutated in cancer (Table S2 in Additional file 1). Targeted capture and PCR amplification were performed as previously described, except that $200 \mathrm{ng}$ of genomic DNA was used for each sample instead of $500 \mathrm{ng}$ [6]. After smMIP capture, amplified products were pooled and sequenced on a single lane of the Illumina HiSeq 2000 platform with paired 100-nucleotide reads and an 8- nucleotide index read.

\section{Primary analysis and variant calling}

Initial analysis steps through to read mapping were performed as previously described [6], except that instead of constructing a consensus read from tagged smMIP molecules, we chose one read per unique molecular tag event at random for subsequent analysis.

Variants were called using SAMtools, and were filtered for positions with phred base quality $\geq 30, \geq 30 \times$ coverage and the absence of a neighboring homopolymer run of four bases or more (Table S2 in Additional file 1). To remove common polymorphisms and enrich for likely somatic mutations, we imposed a number of additional 
requirements, including requiring variants to be observed with an allele balance of at least 5\% within a sample, removing variants present within a modified database of the Exome Sequencing Project [14] and 1000 Genomes [15] pilot project that had first been stripped of all COSMIC variants, removing variants that were present at an allele balance of at least $5 \%$ in two or more control samples.

\section{Copy number analysis}

We compared read depth of smMIP-targeted regions in each tumor against that of the control tissue BI12 to identify high level gene amplifications in tumors. We restricted the copy number analysis to targets with greater than $30 \times$ coverage in control tissue and a GC content ranging from 30 to $60 \%$. To reduce the number of potential artifacts remaining, we removed from consideration (for the purposes of copy number analysis only) 12 genes (AKT1, AKT2, CDK4, CDKN2A, FGFR3, HRAS, KRAS, MYC, NRAS, SRC, STK11, and VHL) that had fewer than 15 probes with sufficient coverage in the control tissue (BI12).

After calculating the ratio of coverage for each probe relative to control tissue from BI12, we used DNACopy [8] to segment genes into discrete levels of coverage and obtain $\mathrm{R}$, the mean ratio of coverage relative to control for each gene. We estimated the copy number for each gene by dividing $\mathrm{R}$ for each gene by the median value of $\mathrm{R}$ across all genes for each tissue. Genes with ratios above 3 were called as amplified and genes with ratios above 6 were called as highly amplified.

\section{Sanger validation}

DNA from five regions of tumor BI09 were subjected to Sanger sequencing (Genewiz, South Plainfield, New Jersey, USA) against positions within IDH1, TP53 and RB1.

\section{Copy number validation}

Tumors with regional heterogeneity in EGFR and PDGFRA detected using smMIP sequencing were confirmed using Taqman qPCR analysis. DNA from each region was analyzed in quadruplicate using commercially available probes against PDGFRA (assay ID: Hs02749151_cn; Life Technologies, Waltham, Massachusetts, USA) and EGFR (assay ID: Hs07526740_cn; Life Technologies). Reference primers amplified a fragment from TERT (number 4403316; Life Technologies). Finally, to compare sensitivity of the smMIP approach, all regions from all tumors were assayed in duplicate for EGFR copy number.

\section{Immunohistochemistry and FISH}

Immunohistochemistry for IDH1 and p53 was performed on 4-micron paraffin sections using mouse anti-human p53 clone (1:2,000 dilution; DAKO, Glostrup, Denmark) and mouse anti-human IDH1 R132H (1:200 dilution;
Dianova, Hamburg, Germany). All tumors were investigated for IDH1 mutation by neuropathology, while only a subset of tumors was investigated for p53 expression by immunohistochemistry (Table S1 in Additional file 1). Immunohistochemistry for EGFR and PDGFRA was performed on 5- to 6-micron paraffin sections using mouse anti-human EGFR, clone 2-18C9 (pharmDx kit, DAKO) and rabbit anti-human PDGFR $\alpha$, clone D1E1E (1:500 dilution; Cell Signaling, Danvers, Massachusetts, USA). Dualcolor EGFR FISH was performed using commercially available probes (LSI EGFR SpectrumOrange/CEP 7 SpectrumGreen, number 32-191053; Abbott Molecular, Chicago, Illinois, USA) with DAPI counterstain using standard methods. Slides were imaged using an Olympus DP72 digital camera mounted on a Nikon E400 microscope. Fifty nuclei were scored for each region. EGFR amplification was called if more than $10 \%$ of nuclei either contained many EGFR signals or exhibited a EGFR:CEP7 ratio greater than $2.1 \mathrm{p} 19 \mathrm{q}$ deletion FISH was performed using commercially available probes (number 04 N60-020, Abbot Molecular) using standard methods.

\section{Data availability}

All sequence data from smMIP capture experiments have been deposited in the NCBI Sequence Read Archive (SRA) under accession number SRP049298.

\section{Additional file}

Additional file 1: Supplementary figures, tables and their associated legends.

\section{Abbreviations}

FISH: fluorescence in situ hybridization; GBM: glioblastoma multiforme; MIP: molecular inversion probe; PCR: polymerase chain reaction; qPCR: quantitative PCR; smMIP: single molecule molecular inversion probe.

\section{Competing interests}

The authors declare that they have no competing interests.

\section{Authors' contributions}

$A K, J M O, R C R$ and JS conceived the study and designed experiments. DLS, AK, MT, AMM, MCS, EG and JRS acquired and processed tissues. JBH, AA, and JOK contributed reagents and analysis tools to the study. AK and EAB performed DNA extraction, MIP capture and PCR validation. $C L$ and AK performed sequencing. MT, LFG and DEB performed immunohistochemistry and FISH. AK performed sequence alignment and mutation analysis. AK, RCR and JS drafted the manuscript. All authors read and approved the final manuscript.

\section{Acknowledgements}

We would like to thank Stephen Salipante and Aaron McKenna for helpful discussions. We would also like to thank the patients and families who have contributed tissue for this project. This work was supported by institutional funds from the University of Washington and the Fred Hutchinson Cancer Research Center the Brain Tumor Research Fund of the Department of Neurological Surgery, and grants from the National Cancer Institute (CA160080 to JS; RO1CA136808 and CA136808 to RCR; R21NS082542 to AMM; CA155360 and (A114567-07 to JO) and the National Institute of Aging (AG039173 to JBH). 


\section{Author details}

'Department of Genome Sciences, University of Washington, Seattle, WA 98195, USA. ²Division of Medical Genetics, University of Washington, Seattle, WA 98195, USA. ${ }^{3}$ Department of Neurosurgery, University of Washington, Seattle, WA 98195, USA. ${ }^{4}$ Clinical Research Division, Fred Hutchinson Cancer Research Center, Seattle, WA 98109, USA. ${ }^{5}$ Department of Pathology, University of Washington, Seattle, WA 98195, USA.

Received: 8 February 2014 Accepted: 4 November 2014

Published online: 03 December 2014

\section{References}

1. Yachida S, Jones S, Bozic I, Antal T, Leary R, Fu B, Kamiyama M, Hruban RH, Eshleman JR, Nowak MA, Velculescu VE, Kinzler KW, Vogelstein B, lacobuzio-Donahue CA: Distant metastasis occurs late during the genetic evolution of pancreatic cancer. Nature 2010, 467:1114-1117.

2. Gerlinger M, Rowan AJ, Horswell S, Larkin J, Endesfelder D, Gronroos E, Martinez P, Matthews N, Stewart A, Tarpey P, Varela I, Phillimore B, Begum S, McDonald NQ, Butler A, Jones D, Raine K, Latimer C, Santos CR, Nohadani M, Eklund AC, Spencer-Dene B, Clark G, Pickering L, Stamp G, Gore M, Szallasi Z, Downward J, Futreal PA, Swanton C: Intratumor heterogeneity and branched evolution revealed by multiregion sequencing. $N$ Engl J Med 2012, 366:883-892.

3. Sottoriva A, Spiteri I, Piccirillo SG, Touloumis A, Collins VP, Marioni JC, Curtis C, Watts C, Tavare S: Intratumor heterogeneity in human glioblastoma reflects cancer evolutionary dynamics. Proc Natl Acad Sci U S A 2013, 110:4009-4014.

4. Snuderl M, Fazlollahi L, Le LP, Nitta M, Zhelyazkova BH, Davidson CJ, Akhavanfard S, Cahill DP, Aldape KD, Betensky RA, Louis DN, lafrate AJ: Mosaic amplification of multiple receptor tyrosine kinase genes in glioblastoma. Cancer Cell 2011, 20:810-817.

5. Nickel GC, Barnholtz-Sloan J, Gould MP, McMahon S, Cohen A, Adams MD, Guda K, Cohen M, Sloan AE, LaFramboise T: Characterizing mutational heterogeneity in a glioblastoma patient with double recurrence. PLOS One 2012, 7:e35262.

6. Hiatt JB, Pritchard CC, Salipante SJ, O'Roak BJ, Shendure J: Single molecule molecular inversion probes for targeted, high-accuracy detection of low-frequency variation. Genome Res 2013, 23:843-854.

7. Turner EH, Lee C, Ng SB, Nickerson DA, Shendure J: Massively parallel exon capture and library-free resequencing across 16 genomes. Nat Methods 2009, 6:315-316.

8. Venkatraman $\mathrm{ES}$, Olshen $\mathrm{AB}$ : A faster circular binary segmentation algorithm for the analysis of array CGH data. Bioinformatics 2007, 23:657-663.

9. Watanabe $\mathrm{T}$, Nobusawa $\mathrm{S}$, Kleihues $\mathrm{P}$, Ohgaki $\mathrm{H}$ : IDH1 mutations are early events in the development of astrocytomas and oligodendrogliomas. Am J Pathol 2009, 174:1149-1153.

10. Lass U, Numann A, von Eckardstein K, Kiwit J, Stockhammer F, Horaczek JA, Veelken J, Herold-Mende C, Jeuken J, von Deimling A, Mueller W: Clonal analysis in recurrent astrocytic, oligoastrocytic and oligodendroglial tumors implicates IDH1- mutation as common tumor initiating event. PLoS One 2012, 7:e41298.

11. Szerlip NJ, Pedraza A, Chakravarty D, Azim M, McGuire J, Fang Y, Ozawa T, Holland EC, Huse JT, Jhanwar S, Leversha MA, Mikkelsen T, Brennan CW: Intratumoral heterogeneity of receptor tyrosine kinases EGFR and PDGFRA amplification in glioblastoma defines subpopulations with distinct growth factor response. Proc Natl Acad Sci U S A 2012, 109:3041-3046.

12. Ino Y, Betensky RA, Zlatescu MC, Sasaki H, Macdonald DR, Stemmer-Rachamimov AO, Ramsay DA, Cairncross JG, Louis DN: Molecular subtypes of anaplastic oligodendroglioma: implications for patient management at diagnosis. Clin Cancer Res 2001, 7:839-845.

13. Okada Y, Hurwitz EE, Esposito JM, Brower MA, Nutt CL, Louis DN: Selection pressures of TP53 mutation and microenvironmental location influence epidermal growth factor receptor gene amplification in human glioblastomas. Cancer Res 2003, 63:413-416.
14. Fu W, O'Connor TD, Jun G, Kang HM, Abecasis G, Leal SM, Gabriel S, Rieder MJ, Altshuler D, Shendure J, Nickerson DA, Akey JM, NHLBI Exome Sequencing Project, Bamshad MJ: Analysis of 6,515 exomes reveals the recent origin of most human protein-coding variants. Nature 2013, 493:216-220.

15. Abecasis GR, Altshuler D, Auton A, Brooks LD, Durbin RM, Gibbs RA, Hurles ME, McVean GA: A map of human genome variation from population-scale sequencing. Nature 2010, 467:1061-1073.

doi:10.1186/s13059-014-0530-z

Cite this article as: Kumar et al:: Deep sequencing of multiple regions of glial tumors reveals spatial heterogeneity for mutations in clinically relevant genes. Genome Biology 2014 15:530.

\section{Submit your next manuscript to BioMed Central and take full advantage of:}

- Convenient online submission

- Thorough peer review

- No space constraints or color figure charges

- Immediate publication on acceptance

- Inclusion in PubMed, CAS, Scopus and Google Scholar

- Research which is freely available for redistribution

Submit your manuscript at www.biomedcentral.com/submit
C Biomed Central 